\title{
Effect of Radiation Models on CFD Simulations of Upward Flame Spread
}

\author{
JIANPING ZHANG, SIAKA DEMBELE, JOHN KARWATZKI, and \\ JENNIFER X WEN \\ School of Engineering, Kingston University \\ Friars Avenue \\ Roehampton Vale, London, SW15 3DW, UK
}

\begin{abstract}
In combustion applications, the weighted-sum-of-grey-gases (WSGG) model is widely used because it is computationally efficient, but it often yields relatively large errors. The objective of this study is to examine the effect of radiation models on CFD predictions of flame spread. To this end, a statistical narrow band (SNB) model and the WSGG model are employed for the simulation of two upward flame spread scenarios, one being a largescale flame spread over a vertical PMMA wall while the other representing flame spread along vertical corner walls. The entire flame spread model also consists of a fourequation turbulence model, an eddy-break-up (EBU) combustion model, a discrete transfer (DT) radiation model, a simple soot model and a non-charring pyrolysis model. Quantitative comparison is made between the prediction results obtained with the SNB model and the WSGG model as well as the experimental data. Results clearly show that the SNB model yields more accurate results than the WSGG approach. However, the SNB model is about four to five times more time consuming than the WSGG model. Therefore, for simulations of complex engineering applications a compromise between accuracy and numerical efficiency should be taken into account.
\end{abstract}

KEYWORDS: upward flame spread, flame spread model, radiation gas model, statistical narrow band model, weighted-sum-of-grey-gases model

NOMENCLATURE LISTING

\begin{tabular}{llll}
$A(i)$ & area of the $\mathrm{i}^{\text {th }}$ burning cell $\left(\mathrm{m}^{2}\right)$ & $x$ & position variable $(\mathrm{m})$ \\
$c_{p}$ & specific heat $(\mathrm{kJ} /(\mathrm{kg}-\mathrm{K}))$ & $y$ & height $(\mathrm{m})$ \\
$f_{v}$ & soot volume fraction & \multicolumn{2}{c}{ Greek } \\
$\Delta H_{c}$ & heat of combustion $(\mathrm{kJ} / \mathrm{kg})$ & $\Omega$ & solid angle $(\mathrm{sr})$ \\
$H_{g}$ & heat of gasification $(\mathrm{kJ} / \mathrm{kg})$ & $\varepsilon$ & surface emissivity \\
$h_{c}$ & heat transfer coefficient $\left(\mathrm{kW} /\left(\mathrm{m}^{2}-\mathrm{K}\right)\right.$ & $\rho$ & density $\left(\mathrm{kg} / \mathrm{m}^{3}\right)$ \\
$I$ & radiant intensity $\left(\mathrm{W} /\left(\mathrm{m}^{2}-\mathrm{sr}\right)\right)$ & $\chi$ & mole fraction \\
$k$ & thermal conductivity $(\mathrm{kW} /(\mathrm{m}-\mathrm{K}))$ & Subscripts \\
$k_{\alpha}$ & absorption coefficient $(1 / \mathrm{m})$ & $b$ & burnt \\
$k_{\text {sca }}$ & soot scattering coefficient $(1 / \mathrm{m})$ & $f$ & flame \\
$L$ & path length $(\mathrm{m})$ & $g$ & gas \\
$p_{g}$ & gas partial pressure $(\mathrm{atm})$ & $\mathrm{ig}$ & ignition \\
$R_{f l u x}$ & radiation heat flux $\left(\mathrm{kW} / \mathrm{m}^{2}\right)$ & $n$ & number of grey gas \\
$s$ & distance in the direction of $\Omega(\mathrm{m})$ & $p$ & pyrolysis \\
$T$ & temperature $(\mathrm{K})$ & $\mathrm{s}$ & soot \\
$t$ & time $(\mathrm{s})$ & $v$ & wave number
\end{tabular}




\section{INTRODUCTION}

A fundamental understanding of the mechanisms by which flames ignite and spread over surfaces of combustible materials is of major importance in fire safety science. In particular, upward flame spread over vertical surfaces is a critical aspect of accidental fires because of its inherent high speed and potential consequences of fire growth to surrounding.

A significant amount of work on the investigation of upward flame spread over solid surfaces has been reported in the literature [1-8]. Three key empirical elements to any model of upward flame spread were identified in [2], namely, the magnitude and spatial variation of the heat flux from the flame to the fuel surface as a function of distance above the pyrolysis front; the height of the flame as a function of the total heat release rate below the flame front; and the dependence of the heat release process from an element of ignited fuel on both external flux and time. Some researchers have attempted to predict fire spread by means of zone and field models, with the flame spread rate incorporated through the use of thermal analysis, empirical formulations, or direct use of Cone Calorimeter data [1-3]. With rapid advancement of CFD method in combustion applications, alternative approaches have been developed where flame spread is governed by a set of partial differential equations that describe and link gas-phase and solid-phase behaviour. By incorporating a simple pyrolysis model within a fire field model, Jia et al. [4], Yan and Holmstedt [5] and Zhang et al. [6] successfully predicted the flame spread phenomenon. The six-flux model was used in [4] to describe the thermal radiation, while a fast narrow band model was used in [5]. This model divides the radiation spectrum and temperature into many intervals and a once-and-for-all database of model parameters was precomputed. This narrow band model offers the advantage of being fast; it was claimed to be more than 20 times faster than the RADICAL model [9]. However, the accuracy of this model is affected by the assumption that the band parameters at the real gas temperature use the preevaluated values at the nearest temperature, and also depends on the temperature resolution to a great extent.

Though much effort devoted, numerical modelling of flame spread still is a highly complicated task due to the complexity of physical and chemical sub-processes involved, including heat transfer, combustion, turbulence, pyrolysis of solid fuels and soot effect. In this study, the RANS based CFD code SOFIE is used as the frame code. A modified fourequation turbulence model, developed by Liu and Wen [10] to emphasise buoyancy effect in turbulence, has been implemented. As surface heat flux has dominant effect on surface flame spread, it is essential to achieve accurate calculations of the combined radiative and convective heat fluxes on the combustible surfaces. The weighted-sum-ofgrey-gases (WSGG) model has been widely used due to its simplicity of implementation and low computational cost, but it lacks the ability of taking into account the spectral dependence of radiative mechanisms and could lead to important errors. In contrast, statistical narrow band (SNB) models describe the radiative properties of gases in terms of mean transmissivities averaged over spectral interval of typically 5 to $50 \mathrm{~cm}^{-1}$ [11]. A formulation of the SNB model based on the Malkmus's approach [12] and employing a literature parameters database has been successfully implemented and investigated by the present authors for pool fires and flame spread studies [6,13], and this approach has shown its ability to yield accurate results.

To examine the effect of radiation models on CFD simulation of flame spread, the SNB and WSGG models are used for the simulation of two flame spread scenarios. One is the large scale PMMA wall fire test conducted by Factory Mutual Research Corporation 
(FMRC) [7]; and the other is an upward flame spread along vertical corner walls [8]. A non-charring pyrolysis model $[5,14]$ is used to describe the pyrolysis of the solid fuel, with a conjugate boundary condition applied to the interface between gas and solid phases. The SNB and WSGG models, along with the pyrolysis model, the four-equation turbulence model, the eddy-break-up (EBU) combustion model, the discrete transfer (DT) radiation method and a soot model are employed for the computations. The simulation results obtained using the SNB and WSGG models are presented and analysed with the experimental data.

\section{MATHEMATICAL MODELLING}

The entire flame spread model consists of the following sub-models: a four-equation turbulence model, an EBU combustion model, a DT radiation model, the WSGG and SNB gas models, a soot model, and a thermal pyrolysis model for non-charring materials. Only brief description of these models closely related to this study is presented here; interested readers may refer to the relevant publications $[10,15,16,20,22]$.

\section{Radiation Model}

Thermal radiation is an important heat transfer mechanism in many combustion problems. Its basic understanding has been well established mathematically as [16]:

$$
\frac{d I}{d s}=-\left(k_{\alpha}+k_{s c a}\right) I+k_{\alpha} E_{g}+\frac{k_{s c a}}{4 \pi} \int_{4 \pi} P\left(\Omega, \Omega^{\prime}\right) I\left(\Omega^{\prime}\right) d \Omega^{\prime}
$$

where $\mathrm{E}_{\mathrm{g}}=\sigma \mathrm{T}_{\mathrm{g}}^{4}$, Stefan-Bolzmann constant $\sigma=5.67 \times 10^{-8}\left(\mathrm{~W} / \mathrm{m}^{2}-\mathrm{K}^{4}\right)$, and $\mathrm{P}\left(\Omega, \Omega^{\prime}\right)$ represents the probability that incident radiation in the direction $\Omega^{\prime}$ will be scattered into the increment of the solid angle $\mathrm{d} \Omega$ about $\Omega$.

The technique employed to solve the radiation transfer equation (RTE) is the discrete transfer model (DTM) developed by Lockwood and Shah [16]. This method involves the tracing of representative rays from one surface to another through the domain of interest. The radiant intensity distribution along each ray is calculated by solving a discretisation of the RTE. The total radiation source constitutes a source term in the enthalpy equation. The DTM solver has been implemented in SOFIE by the code's developers [17].

The WSGG model expresses the total emissivity of a real gas as a weighted sum of grey gas emissivities [18]:

$$
\varepsilon_{g}(T, p)=\sum_{n=1}^{N} a_{n}(T)\left\{1-\exp \left[\left(-k_{g, n} p_{g}-k_{s} \rho_{s} f_{v}\right) L\right]\right\}
$$

The weighting factor, $a_{n}$, and gas and soot absorption coefficients, $k_{g, n}$ and $k_{s}$, are obtained from a fit to total emissivity with the constraint that the sum of coefficients $a_{n}$ is equal to unity. In the present study, the coefficients of Truelove [18] were used with $\mathrm{N}=3$, representing three grey gases.

The SNB model of Malkmus [12] consists in subdividing the spectrum into a small wave number interval $\Delta v$, and then calculating the radiative transfer on each of these narrow bands. The present authors have implemented this SNB approach into SOFIE. The 
spectrally averaged transmissivity of a homogeneous and isothermal gas layer of length $L$ is defined as $[12,19,20]$ :

$\overline{T_{v}}=\frac{1}{\Delta v} \int_{\Delta v} \exp \left(-k_{v} L\right) d v=\exp \left[-\frac{\overline{\beta_{v}}}{\pi}\left(\sqrt{1+\frac{2 \pi \chi p L \overline{k_{v}}}{\overline{\beta_{v}}}}-1\right)\right]$

where $\overline{\beta_{v}}=2 \pi \overline{\gamma_{v}} / \overline{\delta_{v}}$. The mean half width, $\gamma_{v}$, for $\mathrm{H}_{2} \mathrm{O}$ and $\mathrm{CO}_{2}$ is given in [20]. Spectral mean line-intensity to spacing ratio, $\overline{\mathrm{k}_{v}}$, and spectral equivalent line spacing, $\overline{\delta_{v}}$, are functions of temperature and tabulated for a set of spectral intervals [20]. This set of parameters from [20] has been successfully employed by the present authors in previous studies $[6,13]$ and is used in the present study. While using the SNB model, the alternative approach should be sought for the calculation of the transmissivity of soot, and it is calculated using the Mie theory [21]:

$\bar{T}_{v_{\text {soot }}}=\exp \left\{\frac{3}{2} \frac{\pi}{v}\left[\frac{24 \tilde{n} \tilde{k}}{\left(\tilde{n}^{2}-\tilde{k}^{2}+2\right)+4(\tilde{n} \tilde{k})^{2}}\right] f_{v} L\right\}$

where $\tilde{\mathrm{n}}$ and $\tilde{\mathrm{k}}$ are respectively the real and imaginary parts of the soot particle refraction index, and tabulated as a function of the wavelength from $3 \times 10^{-7}$ to $2 \times 10^{-5} \mathrm{~m}$.

In fires, soot can have a very important impact on radiation. The soot volume fraction in Eq 4 is central to the radiation calculation and is provided by the Tesner's soot model [22], embedded in SOFIE and consisting of a physical model for soot formation and an eddy-break-up description for the oxidation process. In this model two additional differential equations for soot mass fraction and soot nuclei number are solved, based on which the soot volume fraction is evaluated as a derived variable.

\section{Flame Spread Model}

A common approach to describe the reaction of the combustible material is to solve the heat conduction equation, using the test data, such as heat release rate from Cone Calorimeter tests, as input. An alternative method is to solve the heat conduction equation and use a pyrolysis model to describe the pyrolysis of the solid fuel. The advantage of the pyrolysis model is that the heat release rate of each solid element is calculated by the model itself, rather than specified by data input. The one-dimensional transient heat conduction equation including the effect of the pyrolysis and charring of the solid fuel can be written as [5,14]:

$\frac{\partial}{\partial t}\left(\bar{\rho} C_{p} T_{\text {solid }}\right)+\dot{m}^{\prime \prime \prime} \Delta H^{*}+\frac{\partial}{\partial x}\left(\dot{m}^{\prime \prime} h_{g}\right)=\frac{\partial}{\partial x}\left(k \frac{\partial T_{\text {solid }}}{\partial x}\right)$

where $\dot{m}^{\prime \prime \prime}=-\frac{\partial \rho}{\partial t}=\frac{\partial \dot{m}^{\prime \prime}}{\partial x}$. The third term on the LHS of Eq. 5 is the energy transfer to or from the vaporised gas as it flows to the solid surface, and will be zero for non-charring materials, and therefore is dropped out in this study. $\Delta H^{*}$ is the heat of pyrolysis, 
representing the energy required to produce the volatiles from the reference state. To determine the heat release rate, the mass loss rate should be evaluated. The overall pyrolysis rate can then be obtained as the summation of mass loss rate of all burning elements, and thus the total heat release rate can be calculated, based on which the flame tip height is obtained using a correlation in [1], and consequently the rate of flame spread can be determined:

$$
\begin{aligned}
& \dot{m}(i)^{\prime \prime}=\frac{h_{c}\left(T_{g}-T_{x=0}\right)+R_{f l u x}-\varepsilon \sigma T_{x=0}^{4}}{H_{g}}, \dot{Q}=\Delta H_{c} \sum_{i}\left[\dot{m}(i)^{\prime \prime} A(i)\right] \\
& y_{f}=y_{b}+k_{f}\left[\dot{Q}_{i g}^{\prime}+\dot{Q}^{\prime \prime}\left(y_{p}-y_{b}\right)\right]^{n}, v_{p}=\frac{d y_{p}}{d t}=\frac{y_{f}-y_{p}}{t_{i g}} \\
& t_{i g}=\frac{\pi}{4} k \rho c\left[\frac{T_{i g}-T_{\infty}}{q_{f}^{\prime \prime}}\right]^{2}, q_{f}^{\prime \prime}=h_{c}\left(T_{g}-T_{x=0}\right)+R_{f l u x}-\varepsilon \sigma T_{x=0}^{4}
\end{aligned}
$$

$\dot{\mathrm{Q}}_{\mathrm{ig}}^{\prime}$ is the energy release rate for the burner which is equivalent to a line-source. Model constants are $\mathrm{k}_{\mathrm{f}}=0.01\left(\mathrm{~m}^{2} / \mathrm{kW}\right)$ and $\mathrm{n}=1$ [1]. Note that, traditionally heat flux $\mathrm{q}_{\mathrm{f}}^{\prime \prime}$ is either specified to be constant, typically equal to 25 or $30 \mathrm{~kW} / \mathrm{m}^{2}$, up to a certain height and then zero above [1] or calculated by means of an empirical correlation [3]. However in the present study it is accurately calculated by the fire field model as shown in Eq. 8.

\section{EXPERIMENTS CONSIDERED AND COMPUTATIONAL DETAILS}

\section{Experiments Considered}

Two experiments are considered. The first one was a previously reported upward fire spread experiment carried out at the FMRC test centre with a 0.025 m thick PMMA slab, $0.58 \mathrm{~m}$ wide $\times 5 \mathrm{~m}$ high [7]. The PMMA wall was extended another $0.3 \mathrm{~m}$ on each side by Marinite panels. At the outer edge of the Marinite panels, a perpendicular $0.6 \mathrm{~m}$ flow barrier was used to minimise the effects of room drafts. On top of the wall, a $3 \mathrm{~m}$ extension provided a background for measuring the flame heights. The ignition at the bottom of the wall was accomplished by $35 \mathrm{ml}$ of heptane in a copper dish. Besides the standard measurements of heat release rate and pyrolysis height, there were six heat flux gauges at various locations on the PMMA panel surface. The second experiment considered was the test carried out by Qian et al. [8]. Here the PMMA samples (0.02 $\mathrm{m} \times$ $0.3 \mathrm{~m} \times 1.6 \mathrm{~m}$ ) were flush-mounted and fixed to large Marinite walls housed in the onehalf scale room corner model with a Marinite ceiling and a Marinite floor (1.6 m high $\times$ $1.0 \mathrm{~m}$ wide $\times 1.0 \mathrm{~m}$ long). The infrared camera was located away from the fire source to acquire the wall temperature through the flame. The PMMA corner walls were ignited at a spot on the corner bottom by a small propane torch. The spread rate of the pyrolysis front region was measured using the IR technique. Heat fluxes at different heights were also measured. A schematic view of both experiments is shown in Figs. 1 and 2. 


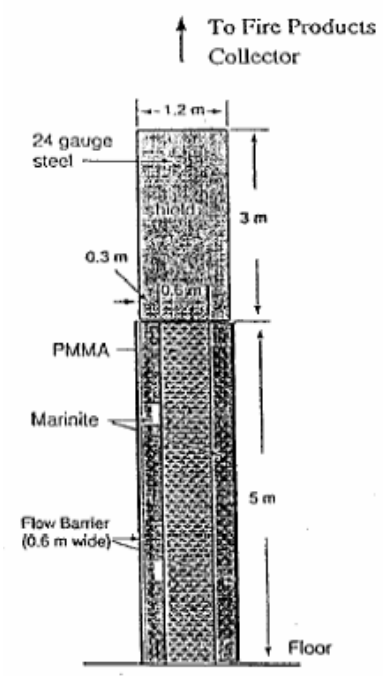

Fig. 1. FMRC PMMA wall test [7].

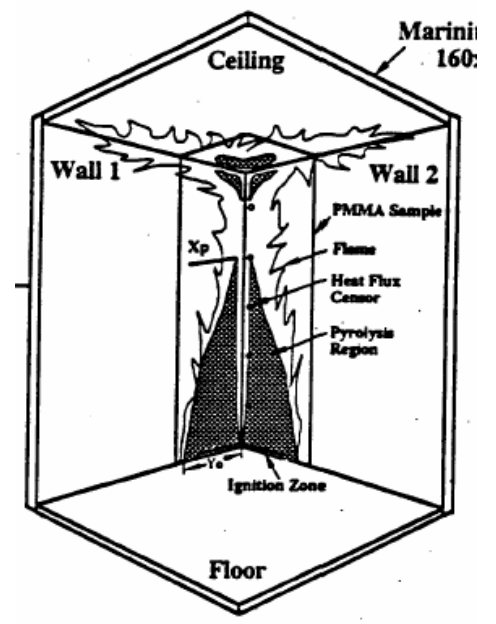

Fig. 2. Qian et al.’s PMMA corner test [8].

\section{Boundary and Initial Conditions}

For the CFD simulation, constant pressure condition is imposed on the open boundary and non-slip condition of velocity is used for the floor. The thermal boundary condition on the floor is adiabatic. The surrounding is air with standard atmosphere pressure of $1 \mathrm{~atm}$ and temperature of $300 \mathrm{~K}$. Uniform inflow velocity is used at the fuel inlet.

\section{Numerical Solution Method}

Due to the symmetric nature of FMRC's test, only half of the fire zone was simulated. The calculation domain was $1.5 \mathrm{~m}$ long $\times 8.0 \mathrm{~m}$ high $\times 1.0 \mathrm{~m}$ wide. A non-uniform $50(\mathrm{X}) \times 100(\mathrm{Y}) \times 40(\mathrm{Z})$ grid was used, with 15 uniform grids across the PMMA board (coarse grid), which implies a cell dimension of $1.67 \mathrm{~mm}$. When solving the onedimensional conduction equation, it is important to examine if the mesh is fine enough to produce essentially grid independent results. To this end, a grid sensitivity test was carried out using 25 uniform grids (fine grid). The relative difference in Fig. 3a is defined as: $\mathrm{Abs}\left(\left(\mathrm{Ph}_{\text {fine }}-\mathrm{Ph}_{\text {coarse }}\right) / \mathrm{Ph}_{\text {fine }}\right)$. As shown in Fig. 3a, only marginal improvement was made; therefore 15 cells are acceptable for the simulation. Grid sensitivity test was also carried out for the flow field. Other two sets of grid number were used, i.e., $60 \times 120 \times 50$ (fine) and $30 \times 60 \times 30$ (coarse). Figure $3 b$ shows the results obtained with the $50 \times 100 \times 40$ grid (medium) are closer to the fine grid, while there is a maximum relative difference of $13 \%$ for the coarse mesh. Note the grid sensitivity tests were only conducted using the WSGG model. For the simulation of Qian et al.'s test, the calculation domain is $1.0 \mathrm{~m}$ long $\times 1.6 \mathrm{~m}$ high $\times 1.0 \mathrm{~m}$ wide. A non-uniform $50(\mathrm{X}) \times 50(\mathrm{Y}) \times 50(\mathrm{Z})$ grid was used, with 15 uniform grids across the PMMA board implying a cell dimension of $1.33 \mathrm{~mm}$. Calculation of radiation is carried out once every 10 -iteration, and the DT rays number used in the present study is $4 \times 16$. The finite control volume method is employed, with the power law differencing scheme, which is first order accuracy. The iterative SIMPLEC algorithm is employed for the pressure correction treatment. The time step used was $1 \mathrm{~s}$ with a total of 1500 seconds physical time simulated for FMRC's test and 500 seconds for Qian et al.'s test. 

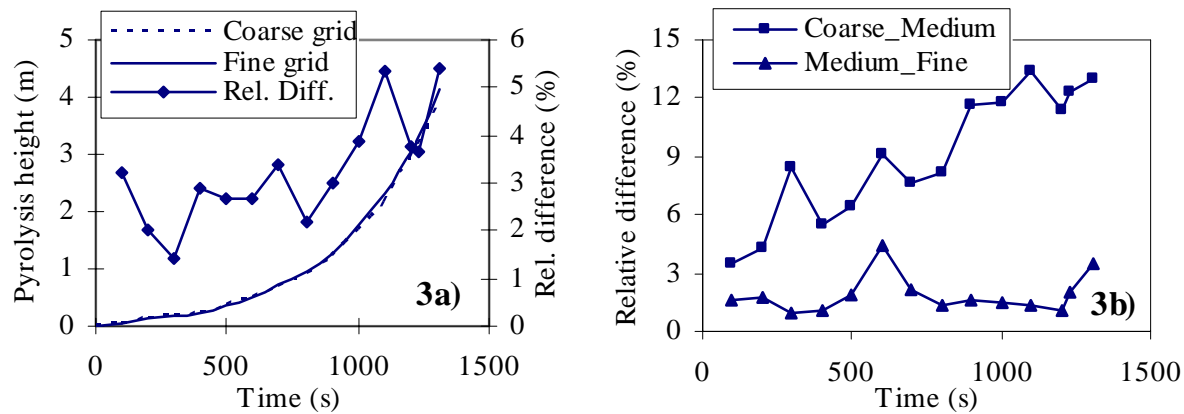

Fig. 3. Grid number sensitivity tests for solid and gas flow fields.

\section{RESULTS AND DISCUSSIONS}

\section{FMRC Case}

Due to the difficulty in simulating the ignition, the experimental heat release rate history is used to synchronise the experiment and the simulation. By matching the initial HRR of $34 \mathrm{~kW}$, the synchronising point is the $350^{\text {th }}$ second or so. The comparison of the predictions of the SNB and WSGG models and the experimental data in Fig. 4 shows a very good agreement up to 750 seconds. After 1000 seconds the WSGG model severely underpredicts the HRR while the SNB model yields fairly satisfactory results. This represents a significant improvement of the SNB model over the WSGG model. The relative difference between SNB and WSGG in this study is defined as: Abs(( $\mathrm{SNB}_{\text {solution- }}{ }^{-}$ $\mathrm{WSGG}_{\text {solution }} / \mathrm{SNB}_{\text {solution }}$ ). The average and maximum relative differences between two models are $15.6 \%$ and $21 \%$, respectively, for HRR predictions. Similarly for the prediction of pyrolysis height, both models perform well up to approximately 750 seconds as shown in Fig. 5. As time increases, there is relatively large discrepancy between the experiment and simulations, especially the results of the WSGG model. At the $1300^{\text {th }}$ second or so, the SNB model predicts the pyrolysis front reaches the top of the board, 50 seconds later than the experiment, while the WSGG model gives a pyrolysis height of only about $4 \mathrm{~m}$. The average and maximum relative differences between SNB and WSGG are $18.1 \%$ and $24 \%$, respectively. These differences will be discussed in the context of the prediction of flow field in terms of temperature and soot volume fraction.

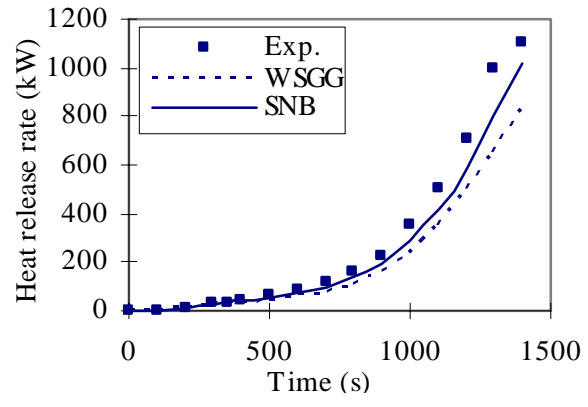

Fig. 4. Heat release rate.

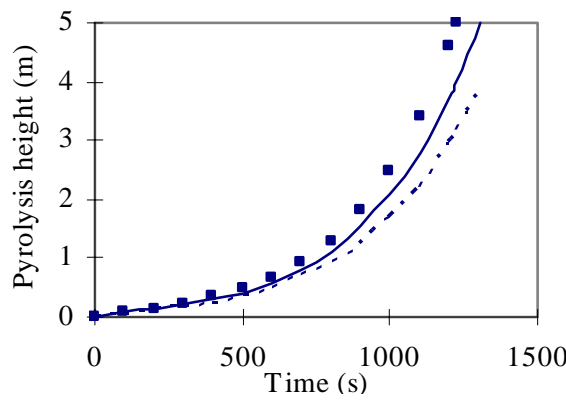

Fig. 5. Pyrolysis height. 
Figures 6a and 6b present the radial profiles of temperature and soot volume fraction at $1200 \mathrm{~s}$ (as shown in Fig. 5 the SNB model predicts a pyrolysis height of about $4 \mathrm{~m}$ at $1200 \mathrm{~s}) 4 \mathrm{~m}$ and $5 \mathrm{~m}$ above the burner. The temperature profiles show the SNB model predicts a higher temperature in comparison to WSGG for both heights, implying a larger flame spread rate. It is worth noting that the temperature is obtained directly from the enthalpy equation using the radiative source term. A good prediction of the radiative source term by the gas model yields more accurate temperatures. Note that the WSGG approach is accurate for homogeneous and isothermal gaseous media. Temperature is relatively constant in the combustible zone. Also the gas absorption in the WSGG formulation is described by absorption weighting factors taken at the emitting body. Since this hot combustion region is a net emitter and when emission (rather than absorption) is the dominant mode, the WSGG model yields predictions closer to SNB. In the region immediately downstream the combustion region, the gas medium is strongly non-isothermal yielding relative larger discrepancies with the WSGG model. Underprediction of the temperature is certainly responsible for the underpredicted flame spread rate and heat release rate. Figure $6 \mathrm{~b}$ shows the prediction of soot volume fraction. At $4 \mathrm{~m}$ the WSGG model predicts higher soot volume fraction than SNB, while a lower value at $5 \mathrm{~m}$. This can be explained by considering the overall distribution of soot volume fraction. In the combustion zone soot volume fraction almost keeps constant just like temperature; into the intermittent region there exists a large temperature gradient, and as temperature decreases to about $800-900 \mathrm{~K}$ soot volume fraction increases to its maximum value at about $1 \mathrm{~m}$ downstream the pyrolysis height location.
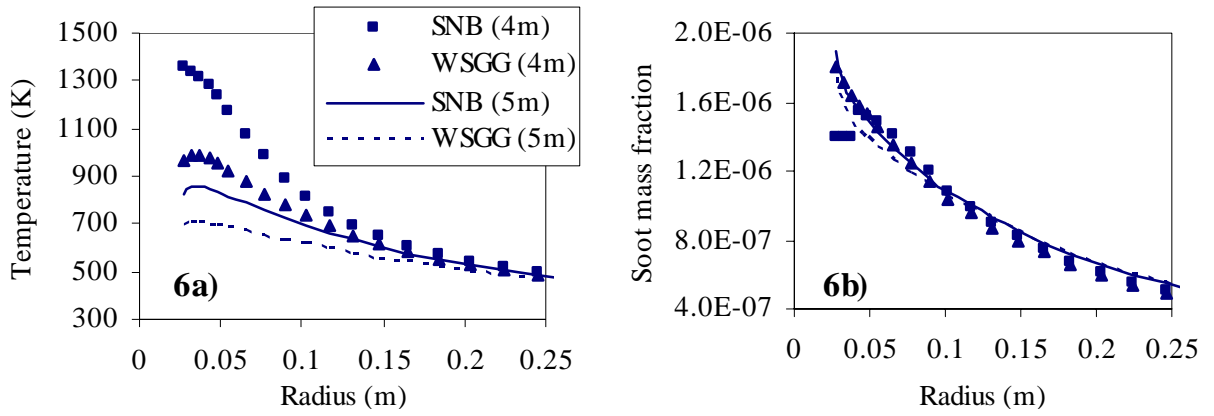

Fig. 6. Temperature and soot volume fraction at $1200 \mathrm{~s}$.

Figure $7 \mathrm{a}$ shows a comparison of the total heat flux between prediction and the experiment at $1200 \mathrm{~s}$. The finding is generally in good agreement with the experimental data. As can be seen, at $1200 \mathrm{~s}$ when the flame reaches the top of the PMMA board, the heat flux almost keeps constant above $1 \mathrm{~m}$ up to $4 \mathrm{~m}$ corresponding to the pyrolysis height for the SNB prediction. At various heights, the measured maximum values for total heat fluxes are within the range of $30-40 \mathrm{~kW} / \mathrm{m}^{2}$, while the predictions give the maximum values about $32 \mathrm{~kW} / \mathrm{m}^{2}$. The underpredicted heat flux could be attributed to the underpredicted temperature by the WSGG model and should be responsible for the underpredicted heat release rate and pyrolysis height previously discussed. Nevertheless, the prediction of the SNB model is closer to the experiment than the WSGG model. The average and maximum relative differences between SNB and WSGG are 9.4\% and 19\%, respectively. Figure $7 \mathrm{~b}$ shows the predicted radiative and convective heat fluxes at 1200 s. As no experimental data are available, the predictions of both models are compared. There is little difference for convective heat flux, while relative large 
discrepancy for radiative heat flux especially at regions the PMMA board is yet to be ignited, because at these regions there exists a big difference for the temperature prediction between the SNB and WSGG models and radiative heat flux is much more strongly dependent upon the temperature.
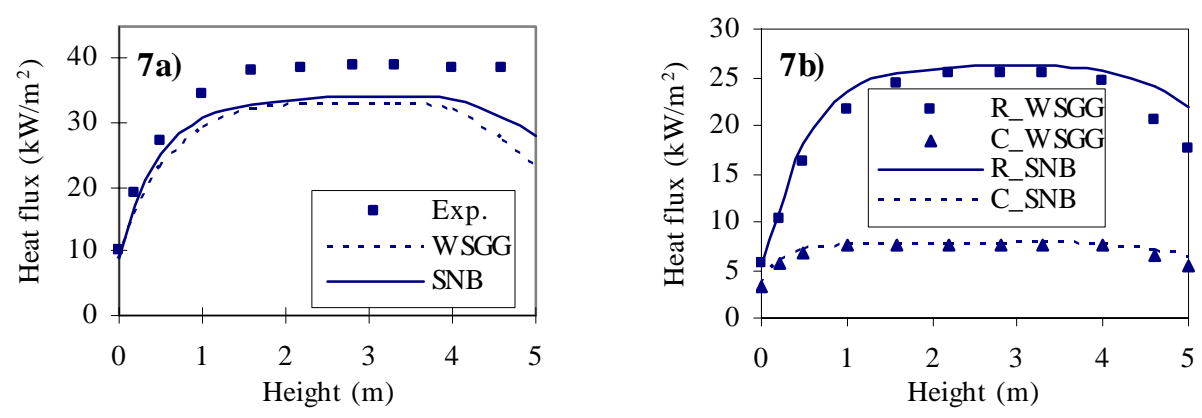

Fig. 7. Total, radiative and convective hat fluxes at $1200 \mathrm{~s}$.

\section{Qian et al.'s Case}

In the experiment, the peak heights of pyrolysis front and flame were detected by IR camera as a function of time. It has been found that the pyrolysis height almost increases exponentially as a function of time. In Fig. 8 the predicted pyrolysis height is plotted as a function of time and compared with the measured data with a reasonable agreement. The SNB model predicts a slightly higher pyrolysis height than the WSGG model, which can be the result that it predicts larger surface heat fluxes. The average and maximum relative differences between SNB and WSGG are $12.8 \%$ and $16 \%$, respectively.

Qian et al. [8] found that the upward flame spread rate for the corner fires is about three times faster than that for the same scale vertical flat wall fire. The high spread rate is mainly attributed to a strong fire induced flow that enhanced heat convection form the flame to the wall surface and an enhanced radiation from the flame to the walls. In order to correctly account for the corner effect on flame spread, the calculation of flame spread rate is slightly adjusted. As the flame height is essential to calculate the flame spread rate, model constant $\mathrm{k}_{\mathrm{f}}$ is modified to a new value of 0.02 . Overall, the SNB model is in closer agreement with the experimental data, though both models underpredict the flame spread rate after about 250 seconds. Alternatively, the correlation $\mathrm{V}=0.0441-\mathrm{y}_{\mathrm{p}}{ }^{0.964}$ [8] can serve well as for the prediction of flame spread rate along the corner as shown in Fig. 9. The average and maximum relative differences are $9.5 \%$ and $14 \%$, respectively.

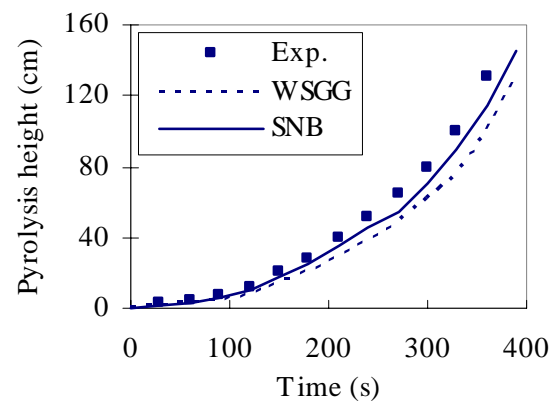

Fig. 8. Pyrolysis rate.

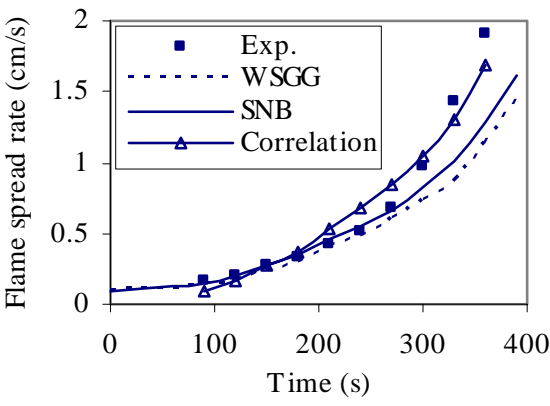

Fig. 9. Flame spread rate. 
Total heat flux received on a PMMA sample surface was measured as a function of time for upwardly spreading fires at five heights along the corner wall. Fig. 10 shows a comparison of heat flux between experiment and prediction at $50 \mathrm{~cm}$ and $100 \mathrm{~cm}$. The overall agreement of the SNB is better than the WSGG model. In the prediction, the heat flux at different heights rapidly increases with time once flame reaches their locations, and thereafter decreases very slowly; however, this peak value was not observed in the experiment. The reason of this could be two-fold. Firstly, convective heat flux decreases as a result of blockage caused by the increasing mass transfer; secondly since radiative heat flux is proportional to the fourth power of temperature, a slight change of the temperature profile after PMMA board was ignited can have serious effect on radiation heat flux. The average and maximum relative differences between SNB and WSGG are $13 \%$ and $23 \%$, respectively.
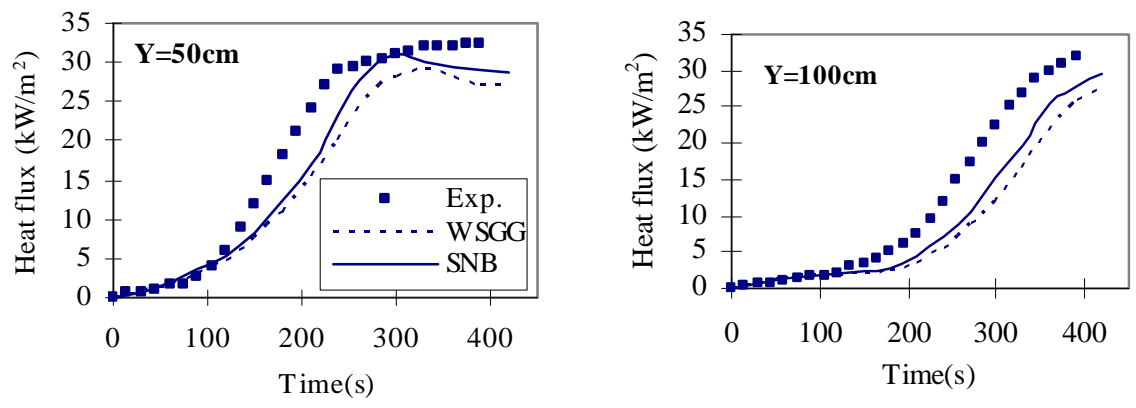

Fig. 10. Total heat flux at $50 \mathrm{~cm}$ and $100 \mathrm{~cm}$.

\section{CPU Time}

The SNB model is significantly more computationally demanding in both cases, since it needs to calculate the transimissivities of the gas mixture for 43 bands, while the WSGG model only needs to calculate emissivities for 3 grey gases. For the calculation of one radiation iteration, the SNB model requires 4-5 times more CPU time than the WSGG model, consistent with the finding in a previous pool fire study [13].

\section{CONCLUSION}

Two radiative gas property models, namely, the SNB and WSGG models, coupled to the discrete transfer method, have been used to simulate upward flame spread over a vertical PMMA surface and along corner walls. The simulations with both models in general compare favourably with the experiments in terms of heat release rate, surface temperature, surface heat flux, pyrolysis height, flame spread rate, gas temperature and soot volume fraction. The following major conclusions may be drawn from the analysis carried out:

1. The WSGG model yields fairly reasonable results, but it is less accurate than the SNB model. Average relative difference between the SNB and WSGG models up to $18 \%$ and local maximum relative difference up to $24 \%$ have been found. Theses differences mainly originate from the differences in predictions for the gas temperature and radiative heat fluxes. The WSGG model could only be used if errors of this magnitude are acceptable. In the mean time, while many uncoupled radiation flow field studies have claimed that the WSGG is largely an inaccurate radiation gas model that should not be employed for real problems, the present study shows when coupled in a CFD code for 
flame spread investigation this approach could yield prediction that agree with the accurate SNB solutions. Therefore, the WSGG model should not be ruled out for practical fire simulations.

2. The SNB model is more accurate, but the computational requirements are also much higher than the WSGG model, up to five times more time demanding. Saving in CPU time can be achieved by introducing a method similar to that in [9] by precomputing a lookup table for transimissivity as a function of temperature and wavelength with the disadvantage of losing in accuracy. A compromise between accuracy and computational efficiency has to be found. Nevertheless the SNB model approach could be used as a benchmark solution in order to assess the accuracy of other simplified gas models such as the WSGG model for a better interpretation of their predictions.

3. Some differences between simulation and experiment have been observed, which could be attributed in part to the complexity and uncertainty involved in the experiment. As mentioned, sub-models can have significant influences on the simulation. Some simple models used in the study could also be responsible in part to the differences. The EBU combustion model is relatively simple. By use of more advanced flamelet laminar combustion models, the prediction of gas temperature and gas fields is expected to be more accurate, which will consequently have positive influence on the modelling of solid phase through the gas-solid interaction.

Further work is to be carried out in terms of reducing computational requirement of the SNB model and coupling this model with some more advanced sub-models for different fire scenarios.

\section{ACKNOWLEDGEMENTS}

This work was supported by Kingston University and Overseas Research Scheme Awards, whom are gratefully acknowledged. The authors also wish to thank Dr. Rubini of Cranfield University for his advice on SOFIE.

\section{REFERENCES}

[1] Quintiere, J.G., "A Simulation Model for Fire Growth on Materials Subject To A Room-Corner Test,” Fire Safety Journal, 20: 313-339 (1993).

[2] Ohlemiller, T. J. and Cleary, T. G., "Upward Flame Spread on Composite Materials," Fire Safety Journal, 32: 159-172 (1999).

[3] Brehob, E. G., Kim, C. I., and Kulkarni, A. K., "Numerical Model of Upward Spread on Practical Wall Materials," Fire Safety Journal, 36: 225-240 (2001).

[4] Jia, F., Galea, E.R., and Patel, K.M., "The Numerical Simulation of the NonCharring Pyrolysis Process and Fire Development within a Compartment," Applied Mathematical Modelling, 23: 587-607 (1999).

[5] Yan, Z. and Holmstedt, G., "CFD Simulation of Upward Flame Spread over Fuel Surface," Fire Safety Science - Proc. of the $5^{\text {th }}$ Int. Symp. 1997, pp. 345356.

[6] Zhang, J., Ferraris, S., Dembele, S., and Wen, J.X., "Numerical Modelling of Flame Spread over A PMMA Surface," $10^{\text {th }}$ International Conference on Fire Science and Engineering, 2004, pp. 1221-1332. 
[7] Wu, P.K., Orloff, L., and Tewarson, A., "Assessment of Material flammability with the FSG propagation model and Laboratory Test Methods," $13^{\text {th }}$ Joint Panel Meeting of the UJNR Panel on Fire Research and Safety, Gaithersburg, 1996.

[8] Qian, C., Ishida, H., and Saito, K., "Fire Growth Models for Materials Upward Flame Spread Along the Vertical Corner Walls,” Technical Report NIST-GCR94-648, National Institute of Standards and Technology, 1994.

[9] Yan, Z. and Holmstedt, G., "Fast, Narrow-Band Computer Model for Radiation Calculations,” Numerical Heat Transfer, Part B, 31:61-71 (1997).

[10] Liu, F. and Wen, J.X., "Development and Validation of an Advanced Turbulence Model for Buoyancy Driven Flows in Enclosures," Int. J. of Heat and Mass Transfer, 42: 3967-3981 (1999).

[11] Liu, F., Gulder, O.L., and Smallwood, G.J., "Three-dimensional Non-grey Gas Radiative Transfer Analyses Using the Statistical Narrow Band Model,” Rev. Gen. Therm, 37: 759-768 (1998).

[12] Malkmus, W., "Random Lorentz Band Model with Exponential-tailed s ${ }^{-1}$ Lineintensity Distribution Function,” J. of the Optical Society of America, 57: 323329 (1967).

[13] Zhang, J., Dembele, S. and Wen, J.X., "Investigation of the Effect of Radiation Models on the Prediction of Pool Fires," $4^{\text {th }}$ International Seminar on Fire and Explosion Hazards, 2003, pp. 303-314.

[14] Lewis, M.J., Moss, J.B., and Rubini, P.A., "Field Modelling of Non-Charring Flame Spread," Fire Safety Science - Proc. of the $6^{\text {th }}$ Int. Symp., Poitiers, France, 1999, pp. 683-694.

[15] Magnussen, B.F. and Hjertager, B.H., "On Mathematical Modelling of Turbulent Combustion with Special Emphasis on Soot Formation and Combustion," Proc. of the $16^{\text {th }}$ Int. Symp. on Comb., 1976, pp. 719-729.

[16] Lockwood, F.C. and Shah, N.G., "A New Radiation Solution Method for Incorporation in General Combustion Prediction Procedures," $8^{\text {th }}$ Symposium (International) on Combustion, 1981, pp. 1405-1415.

[17] Rubini, P., Users Manual of SOFIE (Version 3.0), 1999.

[18] Truelove, J.S., “A Mixed Grey Gas Model for Flame Radiation,” AERE-R8494, 1976, pp. 1-31.

[19] Dembele, S., Zhang, J. and Wen, J.X., "Evaluation of the Correlated-K and other Gas Radiation Models for Combustion Applications,” Fire Safety Science - Proc. $7^{\text {th }}$ Int. Symp., 2002, pp. 16-21.

[20] Soufiani, A. and Taine. J., "High Temperature Gas Radiative Property Parameters of Statistical Narrow Band Model for $\mathrm{H}_{2} \mathrm{O}, \mathrm{CO}_{2}, \mathrm{CO}$, and Correlated-K Model for $\mathrm{H}_{2} \mathrm{O}$ and $\mathrm{CO}_{2}$," Int. J. Heat Transfer, 40: 987-991 (1997).

[21] Bohren, C.F. and Huffman, D.R., "Absorption and Scattering of light by Small Particles,” John Wiley and Sons, New York, 1983.

[22] Tesner, P.A., Snegiriova, T.D. and Knorre, V.G., "Kinetics of Dispersed Carbon Formation,” Combustion and Flame, 17: 253 (1971). 